\title{
Arbor
}

\section{Los estudios de caso en la lógica de la investigación interpretativa}

\author{
José Gutiérrez Pérez, Teresa Pozo Llorente y \\ Antonio Fernández Cano
}

Arbor CLXXI, 675 (Marzo), 533-557 pp.

La investigación interpretativa representa una de las tendencias contemporáneas más reveladoras para el entendimiento del significado de los hechos sociales, culturales, jurídicos, arqueológicos, etnográficos, médicos, psicológicos o educativos. A lo largo de este artículo se ofrece un recorrido panorámico por los procedimientos metodológicos más empleados en el estudio de casos desde la perspectiva de la investigación interpretativa, poniendo énfasis en el proceso, en la logística y en la tecnología de la investigación con datos textuales, imágenes u objetos, procedentes de registros culturales, narraciones orales o documentos institucionales.

Desde un enfoque integrador se presenta una modelización de las diferentes fases de este tipo de investigación: se contemplan los requisitos previos, las eventualidades del trabajo de campo, las estrategias de análisis de los datos, que con mayor asiduidad son empleadas por los investigadores, así como los modelos de difusión pública de la información a que se acogen.

\section{Introducción: Investigación Interpretativa}

Hay problemas de investigación en las Ciencias Sociales, Jurídicas y Humanas que por sus características intrínsecas reclaman un tipo de metodologías alternativas diferentes a los enfoques de la investigación positivista. Estos problemas requieren de la observación persistente y prolongada en los contextos, con el objeto de adquirir una más profunda 
comprensión del fenómeno que se intenta estudiar. Problemas que por su naturaleza demandan de un proceso de investigación no obstrusivo que permita acercarse al corazón de los hechos, a la estructura de los documentos y a la gramática de las fuentes de información primaria con flexibilidad y amplitud de miras.

Es tal la complejidad de dichos problemas que, no pueden ser reducidos a un limitado número de variables, ni siquiera expresarse en forma de hipótesis causales que incluyan aspectos cuantificables. Pensemos en la interpretación de los vestigios de un asentamiento neolítico por parte de un arqueólogo novel; en el estudio de la apropiación lingüística del español por parte de un emigrante subsahariano; en el significado de la catástrofe de Aznalcóllar para un trabajador del lugar; en el diagnóstico pedagógico formulado a partir de una única anamnesis; en las versátiles representaciones infantiles de los modelos científicos; o en la reconstrucción penal que hace un juez de las diferentes versiones de un asesinato,... Son, éstos, problemas de investigación, que se ocupan de los significados singulares, de las vivencias personales, de las influencias del entorno, de las sensaciones, actitudes y sentimientos individuales de sus actores, de la complejidad intrínseca y consustancial a los contextos e interacciones humanas.

Elementos, todos ellos difíciles de expresar en términos numéricos, que han obligado a los investigadores de distintos campos disciplinares a poner a punto una diversidad de estrategias de recogida de datos distintas a los tradicionales arsenales cuantitativos. Estrategias asociadas a un tipo de procedimientos de organización e interpretación también diferentes, que deben adecuarse a la naturaleza misma de esa información: narraciones, imágenes, documentos, enseres, observaciones,...

La Investigación Interpretativa ${ }^{1}$ (desde ahora I.I.) se viene ocupando de estas cuestiones, como un campo joven de indagación interesado por explicar, describir, comprender, caracterizar e interpretar los fenómenos sociales y los significados individuales en la profundidad y complejidad que los caracteriza. También considera los contextos naturales donde se desarrollan y bajo la perspectiva de los intereses, la idiosincrasia y las motivaciones particulares de cada uno de los agentes intervinientes.

La lógica de funcionamiento de este enfoque de los problemas de investigación social y humanística es diferente del resto de las lógicas de investigación tradicionales; tiene sus particularidades y responde a una secuencia procedimental con entidad propia y ampliamente legitimada por la comunidad científica actual. Sin embargo, no se ajusta a una heurística universal que recorra un camino unívoco, aunque esto no nos exime de la necesidad de organizar un marco de principios de procedi- 
miento básicos sobre los que programar una aproximación integradora a un campo fértil y de actualidad. Campo que algunos autores no han dudado en señalar de revolución silenciosa (Denzin y Lincoln, 1994; p. 9): Una revolución silenciosa ha tenido lugar en las ciencias sociales, a lo largo de las dos últimas décadas. Un mestizaje de los límites de las disciplinas se ha producido. Todas las ciencias sociales y las humanidades han volcado su atención hacia un enfoque más cualitativo e interpretativo de la teoría y la investigación... Dicha revolución se refleja en la multitud de manuales, revistas, informes de investigación y reseñas que han sido publicadas en los últimos años.

En la literatura contemporánea conviven opciones metodológicas de corte radicalmente interpretativo, inspiradas en modelos de trabajo que no dan cabida a la más mínima idea de cuantificación, frente a otras visiones más eclécticas que hibridan sus procedimientos con algoritmos y operaciones matemáticas para fortalecer sus principios de cientificidad. ${ }^{2}$

\subsection{Características generales del proceso de Investigación Interpretativa}

El proceso de I.I. está orientado por los intereses del marco de referencia al que se adscribe cada investigador, ya sea la Etnografía, la Etología, la Antropología, la Lingüística, e incluso la propia Ecología Humana. Los enfoques conceptuales de la I.I. vienen determinados por perspectivas teóricas con una amplia tradición disciplinar, que no sólo aportan una metodología, sino también un lenguaje, y una terminología propias. Pero independientemente de la adscripción a una u otra escuela o marco disciplinar, algunas de las características planteadas por Janesick (1994, p. 212), respecto al proceso de investigación interpretativa, son las siguientes:

- El investigador debe permanecer durante un tiempo en el lugar de estudio familiarizándose con el contexto, la sintomatología o las pruebas periciales.

- Es un proceso centrado en lo personal e inmediato que exige una relación directa, cara a cara.

- Se preocupa por la comprensión de una situación social, grupal o individual, y no por la predicción.

- Es un proceso holístico que adopta un enfoque global de la situación y busca la compresión de los hechos en su totalidad, como un retrato global.

- Tal proceso está contextualizado al tener en cuenta las relaciones dentro del sistema social, de la institución o la microcultura. 
- El análisis de la información requiere, al menos tanto tiempo como el consumido en el escenario durante la toma de datos.

- El investigador debe desarrollar una teoría o modelo de lo que ocurre en la situación social. Asimismo, el propio investigador es el instrumento base de la investigación y se requiere poseer las destrezas necesarias para observar y entrevistar.

- Las decisiones deben negociarse respondiendo a cuestiones de tipo ético.

- Incorpora espacios para describir los papeles del investigador, así como la consideración de los posibles sesgos e influencias ideológicas, históricas y culturales.

- Exige un continuo análisis de la información, mediante procesos cíclicos de acercamiento reiterado al contexto y a la información obtenida, que a veces obliga a iniciar nuevos ciclos de toma de datos.

\subsection{La lógica de la investigación interpretativa}

Por lógica de la investigación entendemos todo el conjunto de etapas que ha de recorrer el investigador para alcanzar sus metas; etapas, más o menos comunes, en las que regularmente se suelen amparar mayoritariamente los investigadores para analizar sus datos y formular sus conclusiones.

El sentido en que se utiliza el término dato es más genérico que una simple información numérica: dato cualitativo es aquella información que se obtiene en los procesos de investigación interpretativa, en cuanto información referida a conversaciones, documentos, narraciones, anotaciones de campo, entrevistas grabadas, representaciones, pruebas documentales, enseres de otras civilizaciones... Datos cualitativos pueden ser las cadenas verbales producidas en una entrevista, en una reunión, en un contacto interpersonal, o en documentos escritos, las conductas y sucesos recogidos en las notas de campo. También son datos cualitativos las fotografías, las filmaciones, las grabaciones realizadas en los contextos estudiados y que aportan al investigador información sobre ellos. Los objetos, su distribución, su uso,... constituyen fuentes de datos cualitativos (Gil, 1994; pp. 29-30).

Por tanto, el análisis de estos tipos de información exige un tratamiento adecuado a la naturaleza compleja y a la carga de significación implícita que llevan consigo. Y puesto que los datos cualitativos no son de la misma naturaleza, lógico es pensar que no exista un único método de trabajo en la investigación interpretativa y que sus estrategias de análisis sean variadas. Es más, puede que cada investigador, al margen de asumir los procedimientos de una u otra escuela, teoría, corriente o 
disciplina, se vea obligado en el curso de su trabajo a desplegar, inventar y construir instrumentos o tomar decisiones sobre la marcha que generan novedades importantes en los procesos de planificación, acceso, recogida, análisis y difusión de sus resultados.

El desarrollo de todos estos procedimientos no ha obedecido a un proceso sistemático y bien estructurado por parte de la comunidad científica, sino más bien a un despliegue desordenado de programas de investigación. Cada programa ha ido inventando procedimientos $\mathrm{y}$ construyendo instrumentos en la medida en que, los problemas que aspiraban a abordar y resolver, así lo requerían. En muchos casos, el trabajo ha consistido simplemente en realizar un esfuerzo de adaptación de algunos de los modelos y técnicas de trabajo empleados habitualmente por otras disciplinas; en otros, ha sido preciso improvisar procedimientos inéditos, respaldados muchas veces por los avances tecnológicos disponibles.

Aun cuando existe abundante literatura de investigación sobre este joven campo, en lo que al proceso se refiere, quedan muchos vacíos por cubrir, y queda mucho camino por andar respecto a la modelización de estos procedimientos y la enseñanza, aprendizaje y divulgación de estas estrategias. Actualmente, disponemos también de recursos tecnológicos y software para afrontar los diferentes tipos de datos que resultan de la investigación interpretativa. Ya existe un cuerpo de teoría, más o menos organizada por las diferentes disciplinas, acerca de cómo proceder en cada caso, según que los datos sean imágenes, dibujos, fotografías, registros narrativos, pruebas periciales o registros observacionales.

Sin embargo, aún, la teorización que sobre estos modelos de trabajo se está llevando a cabo, es hoy día insuficiente, y supone un importante reto para los teóricos de la metodología la contemplación, clasificación, revisión y organización de la multitud de casos reales que ya hay disponibles. Por citar algunos ejemplos de las tendencias en el campo educativo, podemos señalar con Bogdan y Biklen (1982, p. 12) como ámbitos de aplicación de la I.I. los tres frentes siguientes:

- El estudio del papel de los diferentes agentes implicados en los procesos educativos tales como padres, profesores, orientadores, alumnos y especialistas en determinados campos.

- Los programas de desarrollo profesional, inserción laboral junto a los procesos de socialización profesional, formación inicial y desarrollo profesional derivados.

- Los procesos de innovación, evaluación e implantación de cambios curriculares y, en general, de reforma educativa. 
La metodología de investigación interpretativa está ampliamente aceptada por la comunidad investigadora. La enseñanza de esta metodología en las Universidades, desde los distintos campos disciplinares (jurídico, antropológico, arqueológico, médico, lingüístico, histórico, sociológico, psicológico, educativo...), se está considerando cada vez más oportuna. No sería aventurado afirmar que, pocas investigaciones contemporáneas del campo social o de las humanidades, se llevan a cabo hoy sin que, de forma exclusiva o complementaria, se incluyan diseños de investigación de esta naturaleza en los que el estudio de caso constituye el recurso metodológico principal de su arquitectura. Hasta hace unos años se trataba de una opción metodológica claramente marginal y con escaso poder de convocatoria. La situación en la actualidad parece comenzar a cambiar (Anguera, 1995a; p. 513). La investigación interpretativa ha alcanzado en esta década un reconocimiento explícito como vía, no única desde luego, para acceder al conocimiento científico (Bartolomé, 1992; p. 15).

\subsection{La complejidad metodológica en la Investigación Interpretativa}

Las dificultades que debe afrontar de cara al futuro este enfoque metodológico no son aún pocas, especialmente en lo que al proceso de investigación se refiere. Denzin y Lincoln (1994, pp. 1-18); García, Rodríguez y Gil (1994, p. 185); Gutiérrez (1999); Hammersley y Atkinson (1994, p. 41-59), Anguera, (1995c, p. 549); Bartolomé et al. (1997, p. 293 294); Velasco y Díaz (1997, p. 89); Coller (2000, p. 53) señalan las siguientes:

- La I.I. constituye un campo muy fructífero, pero muy disperso y bastante heterogéneo, poco organizado hasta el momento y necesitado de aportaciones integradoras y síntesis estructuradas acerca de sus posibilidades y métodos de trabajo.

- Las técnicas son abundantes, aunque no existen reglas fijas para el análisis de los datos, cada investigador avanza y resuelve sus problemas con mayor o menor éxito, pero sin muchas fuentes donde consultar y aclarar sus dudas.

- Estos procesos se apoyan en la intuición, la experiencia, la pericia y la habilidad del investigador para crear, inventar y poner a punto técnicas que le sirvan. La singularidad y privacidad de las técnicas y procedimientos empleados y la falta de detalles con que sue- 
Los estudios de caso en la lógica de la investigación...

len ser descritos los procesos en los informes, hacen que se pierda gran parte de la riqueza que los caracteriza.

- La pluralidad de lenguajes y la diversidad de terminologías al uso, dificultan el intercambio de información y el entendimiento entre los distintos colectivos que investigan bajo esta perspectiva.

- La intransigencia de algunos medios de difusión hace que el impacto de la investigación sea aún muy reducido y bastante restringido a determinados núcleos especializados.

- Las restricciones presupuestarias obligan, con frecuencia, a reducir progresivamente el tiempo de permanencia en el escenario de trabajo, máxime cuando, además, requiere desplazamientos y estancias a tiempo completo. Esto ocasiona que el intervalo de recogida de datos se prolongue y que la investigación se vea afectada por acontecimientos, que no se habían previsto de antemano.

- Las dificultades de formación, en que suelen verse envueltos los equipos, debido a la ausencia de un cuerpo de información organizado, y una falta de tradición en estos procesos determinan que los esfuerzos de trabajo inicial sean más intensos, restándole peso a la propia investigación en aras de una mayor formación.

- La propia realidad impone limitaciones que, a veces, resultan difíciles de abordar y que repercuten a corto y medio plazo en: la mortandad de las muestras, la pérdida de información importante, las trabas para el acceso al campo, la legitimación y el reconocimiento del interés de la investigación, la prohibición de consulta de determinados documentos, los problemas derivados del trabajo en equipo, los conflictos relacionados con la privacidad y la difusión de los datos.

Pese a tal complejidad, el estudio de caso se manifiesta como el método de investigación por antonomasia en esta lógica interpretativa; de ahí, la adherencia de interpretativo al estudio de caso. El estudio de casos es considerado entonces como la estrategia básica para el diseño de la investigación interpretativa. Son muchas las posibles clasificaciones al respecto en este tipo de trabajos, solamente destacamos algunas de ellas: 
CuAdro 1. Tipologías de estudios de casos en la I.I.

\begin{tabular}{|c|l|}
\hline $\begin{array}{c}\text { GUBA } \\
\text { y }\end{array}$ & $\begin{array}{l}\text { Los posibles diseños resultan de una matriz } 4 \times \text { (3 x 2), donde se cruzan las } \\
\text { siguientes variables: [propósitos con que se realiza el estudio de caso } \\
\text { (niveles del estudio x acciones/productos)]. Resultando en realidad 12 tipos } \\
\text { de diseños diferentes, ya que las acciones y productos se refieren a la } \\
\text { misma idea. } \\
\text { Propósitos = hacer una crónica/representar/enseñar/comprobar. } \\
\text { Niveles del estudio = factual/interpretativo/evaluativo. }\end{array}$ \\
\hline $\begin{array}{c}\text { BOGDAN } \\
\text { BIKLEN (1982) }\end{array}$ & $\begin{array}{l}\text { Estudios de Caso Único: Histórico-Organizativo/Observacional/ } \\
\text { Biografía/Comunitario/Situacional/Microetnografía. } \\
\text { Estudios de Caso Múltiple: Inducción analítica modificada (trasferencia a la } \\
\text { generalidad en un mismo contexto)/Comparación Constante } \\
\text { (generalización a contextos diferentes). }\end{array}$ \\
\hline YIN (1984, 1993) & $\begin{array}{l}\text { Los posibles diseños resultan de una matriz (2 x 2) x 5, donde se cruzan las } \\
\text { siguientes variables: [(Caso único/Casos Múltiples x Global/Inclusivo) x } \\
\text { (Exploratorio/Descriptivo/Explicativo/Transformador/Evaluativo)]. } \\
\text { Resultan 20 tipos diferentes de diseños. }\end{array}$ \\
\hline STAKE (1994) & $\begin{array}{l}\text { Estudios de Caso Intrínseco. Se pretende alcanzar la mayor comprensión } \\
\text { posible de un caso concreto. El caso en sí tiene interés. } \\
\text { Estudios de Caso Instrumental. Se persigue con ellos ratificar una teoría o } \\
\text { demostrar algún tipo de intuición o hipótesis. } \\
\text { Estudios de Caso Colectivo. Se indaga sobre un fenómeno o acontecimiento } \\
\text { a partir del estudio intensivo de varios casos relacionados con el mismo. }\end{array}$ \\
\hline
\end{tabular}

La pertinencia del estudio de casos como metodología capital de la I.I. se justifica con los siguientes argumentos:

- Provee densas descripciones que serán esenciales para generar juicios de transferibilidad a otros contextos.

- Proporciona el mejor retrato de situaciones concretas y acontecimientos singulares.

- Responde más adecuadamente a la concepción de múltiples realidades, dando cuenta de las interacciones entre el investigador y el contexto.

- Facilita la comunicación de información con los participantes, favoreciendo el intercambio de puntos de vista y la reconstrucción de significados. 
Los estudios de caso en la lógica de la investigación...

- Permite corregir desajustes entre las perspectivas e interpretaciones del investigador y las visiones de otros agentes participantes.

\section{Fases en el estudio de caso interpretativo}

En términos generales, el desarrollo procedimental del estudio de caso interpretativo [desde ahora E.C.I.] no responde a una estructura lineal y estándar, sino que existen tantas modalidades de trabajo como corrientes y autores han trabajado el tema. Los intentos por generar principios de procedimiento amplios no son nuevos; ya a comienzos del siglo pasado, desde los ámbitos de la Antropología Social se formularon intentos de este tipo bajo una denominación común de Método Comparativo $^{3}$, que en sus diferentes variantes han llegado a nuestros días con aportaciones como las de Glasser y Strauss (1967) y Ragin (1987), preocupados por la construcción de teoría fundamentada. A partir de ese método de la comparación constante, se pueden identificar cuatro momentos básicos:

a) Fase de codificación de los datos en categorías, realizando comparaciones entre aquellos datos incluidos en un mismo grupo, para generar propiedades teóricas de cada categoría. En paralelo, se van formulando las relaciones o hipótesis, que no son otra cosa que resúmenes interpretativos, a la luz de las ideas que van surgiendo de los propios datos.

b) Fase de agrupación e integración de las categorías y de sus propiedades. La comparación sistemática con nuevos datos obtenidos mediante técnicas selectivas de muestreo teórico, permite describir relaciones y nuevos matices dentro de cada categoría.

c) Delimitación de la teoría, a partir de las modificaciones oportunas, motivadas por nuevas comparaciones, se van perfilando progresivamente las relaciones, eliminando aquellas propiedades de carácter secundario, refundiendo categorías repetidas o incorporando nuevas categorías emergentes, hasta conseguir mayor rango de generalización y abstracción de los hallazgos.

d) Explicitación de la teoría retomando de nuevo los datos primarios para documentar, caracterizar y operativizar cada uno de los matices de las diferentes categorías y justificar así las hipótesis o relaciones formuladas.

En esta misma dirección, Goetz y Lecompte (1988, pp. 172-211) reducen a tres estas tareas: procesos de teorización, estrategias de selección secuencial y procedimientos analíticos generales.

a) Procesos de teorización son procedimientos genéricos de análisis y abstracción sobre los cuales se construye y organiza la información a 
partir de estrategias y decisiones de comparación, contraste, agregación, ordenación, determinación de vínculos y relaciones, y especulación.

b) Tareas de selección secuencial son operaciones formales diseñadas para integrar el análisis y la recogida de datos a partir de estrategias como: la selección de casos negativos, la selección de casos discrepantes, el muestreo teórico y la selección de teorías relevantes.

c) Procedimientos analíticos generales son estrategias sistemáticas de manipulación y organización de los datos y conceptos generados en la investigación. Estos procedimientos pueden ser muy variados, desde opciones radicalmente interpretativas con un fuerte predominio de los aspectos semánticos, hasta opciones mixtas donde se hace uso de la estadística textual y de los análisis temáticos.

Inicialmente, el E.C.I. se ha intentado representar con una estructura lineal que comienza en la definición de objetivos, continúa con una recogida de datos, seguida de una fase de análisis y concluye con una formulación de conclusiones. Sin embargo, este tipo de estructura lineal ha sido fuertemente criticado, ya que la investigación interpretativa está sujeta por naturaleza a una estructura circular y recurrente, en la que los análisis exigen volver de nuevo a los datos.

Una y otra vez, el investigador está obligado a interpretar los textos originales para verificar con pruebas positivas o negativas la fortaleza de sus clasificaciones. Como resultado, tal vez se ponga de manifiesto que, es necesario volver a recoger nuevos datos para profundizar en otros aspectos, que en un principio no había considerado, o a los que no se prestó demasiada atención. Estos procesos circulares ocurren también en otras fases de los análisis. Por ejemplo, muchas veces, el investigador, casi sin darse cuenta, comienza a descubrir que las categorías se solapan, se repiten o bien no contemplan aspectos relevantes. Entonces, estas pequeñas crisis obligan a emprender nuevos ciclos de revisión hasta conseguir un marco de categorización potente que resista, contemple e integre la variedad temática incluida en los múltiples textos. Evidentemente, tal tesitura no sería viable bajo modelos de trabajo lineal, en los que no fuese posible acceder nuevamente al campo, para recoger más datos, o bien volver a revisar los textos bajo nuevos criterios de codificación. Tesh (1990) modeliza este proceso desde una secuencia clásica de método científico ajustado a cuatro etapas diferentes: análisis exploratorio, descripción, interpretación y teorización (cuadro 2). 


\section{Los estudios de caso en la lógica de la investigación...}

Cuadro 2. Proceso de teorización en la I.I.

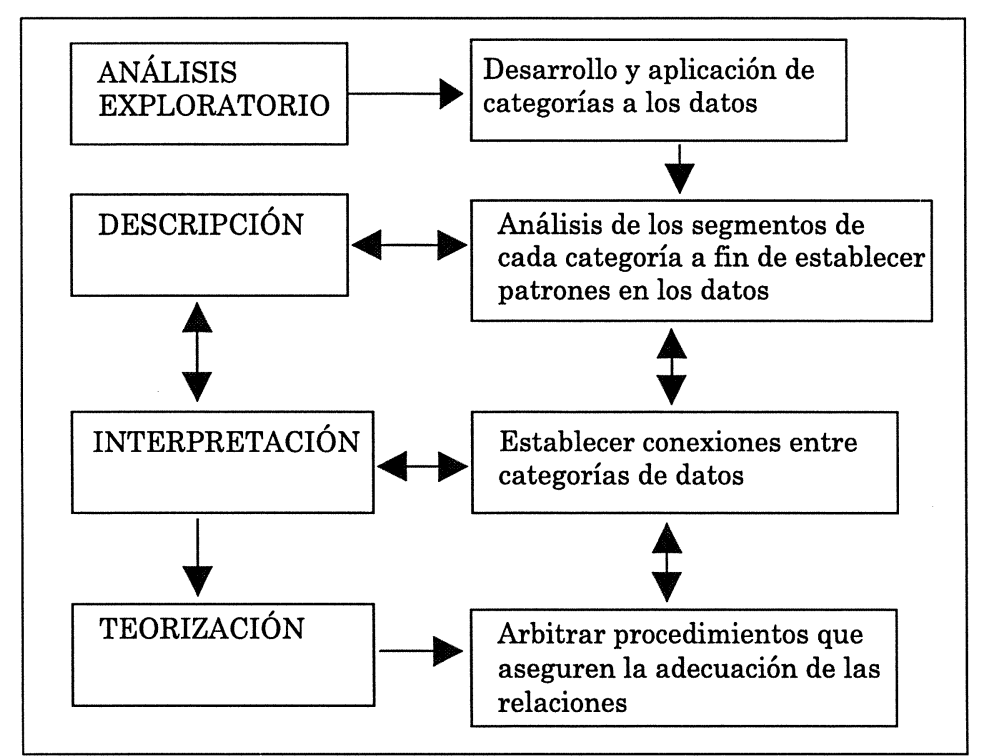

Desde otra óptica, Miles y Huberman (1984), también en Huberman y Miles (1994, p. 429), organizan el proceso en torno a cuatro ideas clave: recogida, organización y reducción de datos, para terminar en la fase de formulación de conclusiones y verificación de resultados (cuadro 3).

CuAdro 3. Modelo interactivo de las fases del análisis de datos

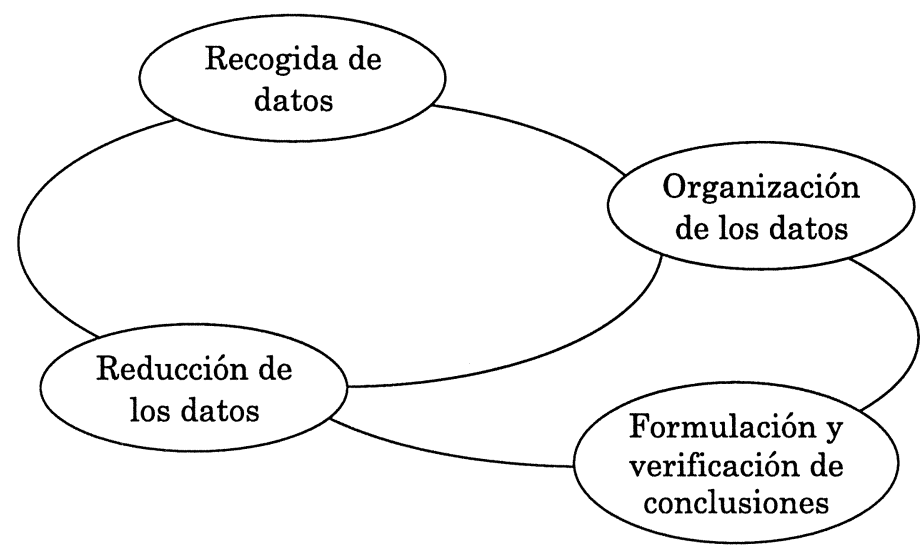


Aun cuando debemos reconocer que no se puede hablar en términos genéricos de un esquema común de E.C.I., en lo que se refiere a la lógica del proceso de la misma, tal y como lo reconocen Lincoln y Guba (1985), Goetz y Lecompte (1988), Tesch (1990), Guba y Lincoln (1994), Hammersley y Atkinson (1994), Maykut y Morhouse (1994), Huberman y Miles (1994), Pérez Serrano (1994), Rodríguez, Gil y García (1996), sí que podemos formular algunos patrones de intersección entre las diferentes corrientes, en cuanto herramienta-guía de orden integrador orientada hacia unas metas comunes: la comprensión de significados, la descripción de subjetividades o la caracterización de contextos. En dicho esquema común, el E.C.I. consta al menos de cuatro hitos básicos: preparatorio, de trabajo de campo, analítico y de difusión de resultados. Desarrollos más pormenorizados del E.C.I. pueden localizarse en Yin (1989), Ragin y Becker (1992), Fernández Cano (1995), Merriam (1998) y Stake (1998).

\subsection{Fase preparatoria del estudio de caso interpretativo}

Es la etapa preliminar del E.C.I. en la que el investigador, a partir de su formación, conocimientos, experiencias e ideología, establece un marco teórico-conceptual que le sirve de referencia para contextualizar y planificar sus actuaciones. Se incluye en esta etapa la revisión documental, la identificación del problema, la propuesta de diseño y acciones a emprender en el curso de la investigación, la delimitación de cuestiones a resolver, la caracterización inicial del escenario en el que se va a trabajar, así como la explicitación del marco teórico.

El prototipo de investigador, que ilustra este enfoque, es el de un naturalista extremadamente meticuloso con personalidad detectivesca, que ocupa su tiempo tomando notas, haciendo dibujos y acopiando todo tipo de muestras, pistas y destalles sobre el caso observado.

\subsection{Fase de trabajo de campo y recogida de pruebas}

Esta fase depende de la formación del investigador en el manejo de determinadas técnicas y de su habilidad para acceder a la información que precisa, para seleccionar el escenario adecuado, elegir informantes clave y conseguir pruebas de interés. Requiere de una toma de decisiones acerca de los procedimientos de almacenaje de los datos. También demanda la puesta a punto de un ensayo piloto para poner a prueba los instrumentos y el diseño, así como decidir la oportunidad de recabar nueva 
información y complementar los muestreos. A través de su habilidad y perspicacia, el investigador obtiene la información necesaria para producir un buen estudio interpretativo; esto destierra algunas creencias acerca de la trivialidad del trabajo de campo, cuyo aprendizaje no requiere de otra destreza que la de ponerse en el lugar de los hechos, e incluso puede llegar a relegarse como una práctica menor en manos de inexpertos: $A$ menudo se oye decir que el trabajo de campo, simple recolección de material en bruto, puede dejarse para los más jóvenes, o bien a personas no especializados, puesto que se reduce a simples técnicas que recogerían esta primera materia prima, sobre la que trabajarían posteriormente los teóricos en la ciudad con imaginación (Panoff, 1975; p. 79).

La vuelta al campo, una vez realizados los primeros análisis, permite ejecutar contrastes sobre las intuiciones y primeras evidencias, así como elegir casos de contraste y afinar mucho más en las técnicas de muestreo más apropiadas.

\subsection{Fase de análisis de datos}

Por análisis de datos interpretativos, entendemos el proceso mediante el cual se organiza y transforma la información recogida por los investigadores sobre un determinado caso, para establecer relaciones, interpretar, extraer significados y sacar conclusiones ${ }^{4}$. El análisis es la fase que sigue a la recogida de datos, y que precede a su interpretación, aunque en investigación interpretativa esto no es literalmente así, argumentan García, Rodríguez y Gil (1994; p. 183): Puesto que en ocasiones junto a las notas de campo y las descripciones suelen aparecer juicios, opiniones, sospechas, dudas, reflexiones e interpretaciones del investigador que alteran este proceso de forma que la interpretación se mezcla con el análisis y la recogida, sin un criterio claro de demarcación entre una y otra fase.

Entre las fases comúnmente admitidas podemos distinguir las cuatro siguientes: reducción de datos; organización, archivo y transformación de datos; obtención de resultados y verificación de conclusiones.

Las decisiones sobre categorización y codificación son las más inmediatas en el proceso general de reducción de datos. En ocasiones, éste suele ser un ciclo que exige una reorganización, revisión y contraste periódico cada cierto tiempo para robustecer y afianzar la consistencia de los cuadros de categorías. Tratándose de material documental, la división de la información en unidades puede realizarse siguiendo diferentes criterios (según se consideren líneas, páginas o bien unidades temporales, unidades gramaticales, unidades de significado,...). 
Este problema ha sido resuelto desde la perspectiva del análisis de contenido por Sánchez Carrión (1985), Bardín (1986), Krippendorf (1990), Pérez Serrano (1994), Anguera (1995b), con la diferenciación entre unidades de contexto (que corresponden a las unidades de análisis) y unidades de registro (que son los fragmentos más pequeños de texto con significación propia dentro de cada unidad de contexto). La identificación y clasificación de unidades, a partir de la elaboración de sistemas de categorías exhaustivas y mutuamente excluyentes, seguidas de procedimientos de agrupamiento, operativización y reestructuración, son actuaciones necesarias en esta etapa.

Una categoría es una unidad de sentido que justifica el reagrupamiento de varias observables. La categoría resulta de un dimensionamiento de la realidad o aclara una idea directamente ligada a un concepto. Por categorización entiende Gil (1994, p. 535): el proceso que trata de romper el corsé del rigor matemático en busca de una simplificación de la realidad que respete un cierto grado de complejidad estructurada. La descontextualización es un primer paso del camino que lleva, por medio de la segmentación/categorización, desde una complejidad excesiva a un modelo con una complejidad manejable.

Las tareas de disposición, organización y transformación de los datos en tablas de contingencia y tablas de incidencia, o bien mediante sistemas de representación gráfica convencional o diagramas creativos, junto al manejo de redes semánticas y mapas conceptuales, pueden ayudar al investigador en esta fase de interpretación de los datos.

Las tareas de obtención de resultados y verificación de conclusiones implican el uso de metáforas y analogías. La inclusión de fotografías, representaciones o viñetas donde aparezcan fragmentos narrativos e interpretaciones tanto del investigador como de otros agentes, constituyen procedimientos útiles para esta fase que culmina con las oportunas estrategias de triangulación, auditoría y validación cara a cara con otros miembros del equipo y otros agentes del contexto.

\subsection{Fase de difusión de resultados}

En esta fase deben considerarse cuestiones importantes como: las características de la audiencia a quien va dirigido el informe, el estilo literario del informe, la validación y legitimación pública del mismo y las opciones de difusión y comunicación pública de los hallazgos obtenidos.

El carácter abierto y cambiante de los E.C.I. obliga a los investigadores a emplear grandes esfuerzos en la redacción de los informes. De he- 
Los estudios de caso en la lógica de la investigación...

cho, se suele admitir que la estructura libre de estos trabajos es la que mayor dosis de creatividad e imaginación requiere, a la hora de presentar y organizar los datos y las evidencias que de ellos se derivan.

Un elemento decisivo de esta fase son los destinatarios, esto acarrea diferentes adaptaciones de ese informe final en orden a las características de la audiencia; adaptaciones en lo referente a estilo, vocabulario, contenido, orden de presentación de la información y detalles de la misma. Entre las posibles audiencias receptoras de un informe de esta naturaleza, podemos señalar: los patrocinadores (Administración Pública, entidades bancarias, fundaciones,...), que con sus fondos, recursos y apoyos hacen posible la investigación; la comunidad científica, formada por colegas universitarios, investigadores y expertos en esa parcela de conocimiento; sectores sociales con intereses en esa información (políticos, administradores, sectores profesionales...); los agentes de la propia comunidad donde se ha llevado a cabo la investigación.

Conseguir la aceptación por credibilidad de un informe es, en parte, un logro intelectual y, en parte, una habilidad para comunicarse y tratar con superiores, colegas, instituciones y personas, que ejercen algún tipo de poder sobre la carrera del investigador, o tienen algún vínculo con la investigación. En el sentir y actuar ético de cada investigador reside el que esa tensión sea más un acto intelectual y un proceso de comunicación fluido, que una pura «maniobra» dirigida exclusivamente a recabar más fondos para el proyecto, a complacer a la audiencia receptora o a convencer a la jerarquía que ha de supervisar dicha investigación.

Entre las posibles situaciones de interacción que puede generar un informe con sus potenciales audiencias locales, Erickson (1989; p. 284-286) señala cuatro patrones típicos, que resultan del cruce entre dos variables de interés: a) la diferenciación entre información conocida e información desconocida; y b) la distinción entre aquella información que al conocerse llegaría a ser positivamente valorada y aquella que podría despertar cierto recelo, suspicacia o incluso reacciones negativas por parte de los potenciales lectores locales. De la combinación de estas opciones resultan cuatro tipos de información diferentes:

Información tipo 1. Es aquella información incluida en el informe, que ya conocían de antemano algunos o todos los miembros locales, y provoca una valoración positiva o neutra, o al menos, no despierta ningún tipo de reacciones en contra por parte de la audiencia.

Información tipo 2. Es aquella que ya conocen todos o algunos miembros de la comunidad local y que podría despertar en todos o algunos miembros de la comunidad un cierto rechazo al ser difundida. Ésta es, sin duda, la información más delicada de presentar en un informe dirigido a 


\section{José Gutiérrez Pérez, Teresa Pozo Llorente...}

un público local. Una información de esta naturaleza podría provocar serias reacciones de incomodidad o indignación en todos o algunos miembros del centro o comunidad, por tratarse de aspectos que afectan de forma muy directa a intereses, hábitos o conductas de determinados miembros.

Información tipo 3. Es aquella información desconocida para todos los miembros de la comunidad local en que se ha llevado a cabo la investigación, y que de conocerse sería muy bien recibida. Éste es el tipo de información que menos problemas plantea, y la que más le complace recibir a los diferentes agentes participantes en un proceso de indagación prolongada, ya que supone un refuerzo positivo y aporta un interesante elemento de reflexión sobre aspectos que pasaban desapercibidos a los miembros de la comunidad.

Información tipo 4. Es aquella que se desconoce y que podría despertar un cierto rechazo o actitud negativa por parte de la comunidad local. Debe ser manejada con sumo cuidado en los informes, aunque su impacto negativo, al ser desconocida, puede no ser tan intenso como la tipo 2, si bien puede afectar a la autoestima de ciertos individuos y grupos del contexto local.

En definitiva, los informes de E.C.I., como consecuencia de la naturaleza de la información que manejan, deben poner especial atención a la diversidad de intereses personales e institucionales que entran en juego, especialmente, en el seno interno de las comunidades locales. Por tanto, deben estimarse de antemano los posibles impactos que pueda causar la divulgación de una determinada información en las diferentes sensibilidades de los agentes de la comunidad local. El investigador interpretativo puede llegar a ejercer una poderosa influencia, sobre los individuos y los contextos en los que desarrolla su trabajo, ya que los resultados de su investigación pueden contribuir a estimular procesos de reflexión individual o colectiva que finalmente pueden originar transformaciones, cambios y decisiones de cierta trascendencia.

Por estas razones, es usual que de un E.C.I. se deriven diferentes tipos de informes. En concreto, se suelen distinguir, al menos dos tipos con sus adaptaciones según los destinatarios (Denzin, 1994; p. 501). El informe de caso interpretativo provisional (documento privado que se diferencia de las anotaciones o datos del cuaderno de campo por el grado de elaboración y la búsqueda de conexión entre los datos), y las diferentes versiones de informe de caso final, según los destinatarios, respaldado por la legitimidad que otorga la negociación del documento con otra serie de agentes (por lo general, colegas o investigadores colaboradores), y el carácter público que va adquiriendo al ser criticado, censurado, revisado, reescrito y asumido por otros miembros de la comunidad implicados en el proceso de investigación. 
Los estudios de caso en la lógica de la investigación...

Tras sucesivos ciclos de negociación, intercambio y lectura compartida de borradores, el informe de un E.C.I. va ganando en estilo, comprensión, ética y disponibilidad para su divulgación e incluso publicación.

$\mathrm{El}$ informe escrito de un E.C.I. no es el único canal posible para dar a conocer el resultado de un estudio de esta naturaleza; a veces, la transmisión oral o la presentación bis a bis de los hallazgos, puede ser la mejor forma de dar a conocer los resultados de un estudio. La transmisión oral es una de las mejores estrategias para validar la calidad de las evidencias, especialmente cuando los destinatarios son el público local con el que se ha desarrollado la investigación.

La comunicación de los resultados de la investigación, a la comunidad de investigadores o a otras audiencias potencialmente interesadas en el tema, es una etapa tan importante como las anteriores. No tendría sentido todo el trabajo previo si al final no se diesen a conocer los resultados. La ciencia se valida a sí misma por la capacidad de soportar y resistir ante las críticas de la comunidad, sin esas críticas no llegaría a legitimarse como tal y nunca podrían tener continuidad los trabajos de investigación. El valor de la investigación está en razón directa con las personas que lo leen. Una investigación que no se comunica, bien como memoria, conferencia, artículo o cualquier otro medio, no contribuye a aumentar el cuerpo de conocimientos teóricos y, por lo tanto, su valor es nulo (Colás, 1994). Pero, además, si los resultados no se comunican de forma efectiva, no serán tenidos en cuenta. El investigador, y nadie más que él, es el responsable de la eficacia de la comunicación: sólo él será el culpable si nadie puede entender lo que ha hecho o juzgar su valor adecuadamente; la claridad, la sencillez de estilo y la brevedad son virtudes capitales.

Antes de elaborar los informes es importante realizar las adaptaciones oportunas, según el tipo de público al que éstos vayan destinados. No es lo mismo un informe dirigido a una revista de educación, cuyos lectores potenciales serán profesores de universidad o investigadores educativos, que dirigirlo a un boletín informativo de carácter educativo destinado a profesores y equipos directivos de centros educativos, que dirigirlo a un periódico; ni tampoco es lo mismo dirigirlo a la institución que patrocina y subvenciona el estudio, que dirigirlo al grupo de centros, padres o colectivos que participaron en el trabajo. Para cada audiencia, el informe del caso se realiza con la finalidad de cumplir unos requisitos determinados. Cuando los informes aspiran a ser editados en forma de libro artículo o comunicación, deben ajustarse a una serie de patrones y manuales de estilo. El manual de estilo más ampliamente utilizado por la I. I. es el propuesto por la Universidad de Chicago (1993), y recomendado por asociaciones como la A.A.A. (American Anthropological Association). 


\section{Criterios de validez del estudio de caso interpretativo}

A diferencia de la metodología positivista, que presume de validez interna y externa, la I.I. prefiere hablar de una validez epistemológica, validez de consenso o validez cara a cara; es decir, sus fuentes de credibilidad están suficientemente garantizadas cuando: el texto está correctamente triangulado, basado en indicadores naturales, adecuadamente adaptado a una teoría, es comprehensivo en su enfoque, creíble en sus mecanismos de control utilizados, consistente en los términos de su reflexión, y representa de forma profunda, clara y completa las características del contexto y el sentir de los otros (Ruiz, 1996; p. 219), minimizando al máximo la pérdida de información relevante.

Los criterios de verdad de un E.C.I. residen en la selección de aquellas construcciones que resulten mejor informadas, más documentadas y sobre las que se obtenga mayor consenso, en función de su adecuación a los datos y a la información disponible. Por tanto, la calidad de un estudio de caso no puede establecerse en orden a los clásicos criterios de validez y fiabilidad, más bien debe sustentarse sobre criterios propios; esto es, en criterios tales como: el valor de verdad, la aplicabilidad, la consistencia y la neutralidad (Lincoln y Guba, 1985; p. 85-86):

CuAdro 4. Criterios de la calidad de la I.I.

- El valor de verdad/credibilidad, se refiere a la credibilidad y confianza que ofrecen los resultados de la investigación, basándose en su capacidad explicativa ante casos negativos y en la consistencia entre los diferentes puntos de vista y perspectivas. Es decir, al isomorfismo que se establece entre los datos recogidos y la realidad.

- La aplicabilidad/transferencia, se refiere a la posibilidad de transferir los resultados obtenidos en ese contexto a otros contextos de similares condiciones, bajo una situación de investigación en idénticas condiciones.

- La consistencia/dependencia, se refiere a la posibilidad de replicar el estudio y obtener los mismos hallazgos.

- La neutralidad/confirmabilidad se refiere a la independencia de los resultados frente a motivaciones, intereses personales o concepciones teóricas del investigador. Es decir, garantía y seguridad de que los resultados no están sesgados. 
Los estudios de caso en la lógica de la investigación...

Se requiere de estrategias complementarias de triangulación (triangulación de técnicas, de agentes, de tiempos, e incluso triangulación metodológica) y de la puesta a punto de procesos de auditoría interna y externa (basada en la comprobación de las inferencias y en la evaluación del diseño por parte de agentes internos-participantes o por expertos, externos al lugar donde se desarrolla la investigación). Todo ello permitirá documentar la información y contrastarla según diferentes puntos de vista.

Entre las estrategias más usuales que suelen emplearse para dar calidad a un estudio de caso interpretativo, en orden a garantizar el rigor y la confianza de los hallazgos que de él se derivan, destacamos según criterio:

- Respecto al criterio de credibilidad: uso de observación persistente, comentario de pares y comprobación con los participantes. Además, procedimientos de triangulación y acopio de material de contraste documental.

- Respecto a la transferencia: uso de muestreo de máxima variabilidad, descripciones en profundidad y diversificación en las técnicas de recogida de información.

- Respecto a la dependencia: empleo de auditoría y control de calidad externa de la información y procedimientos de réplica en la verificación de conclusiones.

- Respecto a la confirmabilidad: utilizando la comprobación externa de conclusiones, fidelidad al dato directo mediante transcripciones textuales, fotografías, citas y referencias directas; así como, también, transparencia en los supuestos epistemológicos y metodológicos empleados.

\section{Sugerencias para una logística en el Estudio de Caso Interpretativo}

El desarrollo de programas informáticos adecuados, para el almacenamiento, manejo, búsqueda, codificación, organización, clasificación, análisis de datos y edición de informes ha supuesto una importante ayuda para el avance y desarrollo de los E.C.I. La Informática, fundamentalmente, ha liberado al investigador de una serie de tareas artesanales, que le obligaban a ejercer de coleccionista y documentalista a la vez, invirtiendo una fracción importante de su tiempo en actividades meramente mecánicas, pero con suficiente trascendencia como para afectar a la calidad final del producto. El manejo físico de los datos una vez recogidos ha sido una tarea tan importante y no menos complicada que las 
restantes fases del proceso. Hasta hace apenas unos años, se consideraba un indicador de éxito en la investigación la propia habilidad de los investigadores para archivar y manejarse con meticulosidad, fluidez y orden en las montañas de datos con que trabaja la investigación interpretativa.

Además, junto al factor abundancia hay que situar el carácter recurrente propio de la lógica de la investigación interpretativa: los datos cualitativos han de ser leídos y revisados en sucesivas ocasiones, ordenados, clasificados de acuerdo con los temas o conceptos a los que alude su contenido, y periódicamente sometidos a comprobaciones y contrastes. Esto ha obligado a los investigadores a disponer de estructuras físicas de almacenaje convencional en fichas, sobres, archivos y soportes de clasificación dinámicos y de fácil consulta. Es necesario, por tanto, disponer de recursos que agilicen el reiterado acceso, permitan una recomposición periódica, el traslado de un lugar a otro, la reorganización cada cierto tiempo, su agrupamiento y desagregación, así como su clasificación según criterios alfabéticos, numéricos, cronológicos, por colores y por contextos.

Cada investigador, en definitiva, ha desarrollado sus propias artimañas para manejarse con los ingentes volúmenes de datos que se generan en la investigación interpretativa. Cuando deseo intentar la escritura del primer borrador de un capítulo completo, empiezo a trabajar en el suelo. Las categorías que se van a destinar a un capítulo en particular pueden ser así movidas de un lado a otro como un montón de tarjetas hasta que se llega a un fluido narrativo lógico y sensato. Primero esto, luego eso, a continuación aquello (Ball, 1995; p. 223).

Pero además de amplias superficies, donde desplazar y mover físicamente los datos, carpetas, sobres, ficheros, archivadores, vitrinas, tijeras, lápices de colores y etiquetas constituyen literalmente las herramientas y arsenales más usuales. Primeramente, sugieren Taylor y Bogdan (1984, p. 168): se recortan las notas de campo, las transcripciones y otros materiales y se colocan los datos de cada categoría en carpetas de archivo o en sobres de papel. Luego, señala Ball (1995, p. 222): pongo en una misma carpeta un conjunto de sobres con datos en su interior y otro conjunto de notas analíticas y comentarios interpretativos. El investigador debería, entonces, disponer de una serie de carpetas que documenten su trabajo. Cualquiera de estas carpetas podría contener fotocopias, fotografías, cintas de audio o vídeo, recortes de publicaciones, notas escritas o documentos oficiales. Los títulos y contenidos de las carpetas podrían cambiar con el transcurso del tiempo.

Incluso se recomienda escribir en la propia portada de las carpetas una breve descripción del contenido de cada categoría, acompañada de 
una selecta elección de aquellas citas literales que mejor representen el campo semántico que cubre dicha categoría; eso facilita enormemente la redacción de los informes y el manejo de los datos.

Junto a las carpetas, el uso de ficheros ha sido otra de las herramientas más empleadas. Cada unidad codificada podía ser trasladada a una ficha, y el conjunto de fichas podría ser organizado por temas dentro de un fichero. La aparición de la fotocopiadora, agiliza bastante esta tarea de elaboración de ficheros, evitando los esfuerzos de duplicación manual de aquellos fragmentos de texto que, al contener más de una categoría, debían clasificarse en varias fichas. Se han llegado a distinguir dos tipos diferentes de fichas: aquellas que simplemente transcriben literalmente los fragmentos de texto y aquellas que, además, incorporan una numeración de las líneas de texto y breves comentarios o memorandos al lado de cada fragmento, indicando posibles relaciones con otras categorías.

Sin lugar a dudas, el gran avance en la mecánica y la artesanía del trabajo con los datos (lo que aquí hemos venido a denominar como logística) y la elaboración de informes se ha producido con la llegada del ordenador y la impresora conectados al vídeo y cámara de fotos digital, al menos en lo que respecta a estrategias de almacenamiento, organización, clasificación, búsqueda y recuperación de datos. El ordenador puede sustituir de manera satisfactoria a herramientas como: lápices de colores, fotocopiadoras, tijeras, cola de pegar o fichas, ganando claramente en eficacia cuando se trata de ordenar o recuperar los fragmentos de texto (Rodríguez, Gil y García, 1996; p. 240). Pero, tenemos la melancólica impresión que, aquellas «mágicas» expectativas, que despertó la computadora desde el comienzo de la década de los ochenta para la realización de E.C.I., aún no han llegado a conseguirse plenamente, dado que el ordenador no ofrece soluciones automáticas a los problemas planteados en el análisis interpretativo (Ruiz, 1996; p. 316).

Los programas de análisis de datos cualitativos sirven solamente para sistematizar y controlar el procesamiento y el análisis de los datos, pero será el investigador quien debe asignar significados a esos datos antes y después de los análisis (Huber, 1988; p. 84; Rodríguez et al. 1995).

Reconocer las limitaciones de la propia tecnología constituye un primer paso para poder aprovechar sus múltiples posibilidades. El ordenador ofrece una valiosísima ayuda en el tratamiento y manejo de textos y fotografías, en el reconocimiento de palabras, en la agregación y separación de unidades, en la estimación de distancias, en el conteo de frecuencias, en la verificación de jerarquías (Tech, 1990; p. 150), e incluso puede favorecer enormemente la construcción de teoría fundamentada con la si- 
mulación de modelos de inteligencia artificial. Pero la consecución de algoritmos y procedimientos tecnológicos, que se acerquen a la formidable complejidad de los sistemas semánticos humanos, es un reto aún por alcanzar. Hay tareas que son intrínsecamente humanas tales como el descubrir significados compartidos, el reconocer y elaborar categorías o el construir interpretaciones globales sobre el mundo y las concepciones que la gente posee sobre el mismo.

El papel del ordenador es positivo, si contribuye con su velocidad y capacidad de almacenamiento a la investigación. Pero si el manejo de su tecnología absorbe parte de las energías y desvía la atención a problemas tecnológicos más que interpretativos del propio texto, sus enormes posibilidades se verán apagadas por sus amplias limitaciones en el desarrollo y resolución de los problemas educativos. ¿Serán capaces los ordenadores del futuro de ir más allá de recuperar palabras, identificar formas, reconocer colores o contar sílabas en los procesos de análisis de textos e interpretación de sus significados literales o metafóricos? Mientras tanto, afortunadamente, será el cerebro del investigador el que interprete, busque relaciones y dé sentido a los datos.

\section{Notas}

${ }^{1}$ Hemos empleado el término Investigación Interpretativa, en lugar de Investigación Cualitativa, por considerarlo el más inclusivo de todos ellos, además por eludir la connotación de contrario a lo cuantitativo y por integrar todo el conjunto de enfoques de la investigación observacional (Erickson, 1989; p. 196). También, esta acepción de interpretativo permite diferenciarlo debidamente de otros enfoques cualitativos más orientados al cambio y la toma de decisiones, como son los distintos tipos de Investigación-Acción. Tampoco nos hemos cerrado a una visión parcial de un tipo de investigación interpretativa, derivada de una escuela de pensamiento en concreto (como pudiera ser la Fenomenológica, el Interaccionismo Simbólico, la Etnometodológica, la corriente de las Teorías Fundamentadas o la propia Metodología Observacional), hemos preferido en su lugar optar por una visión integradora y global común a este cruce de enfoques teóricos y metodológicos, tal y como lo proponen Denzin y Lincoln (1994, pp. 3-4) o Walford (1995).

2 Aun a costa de perder profundidad en algunas cuestiones, hemos preferido organizar el tema desde una visión integradora que aglutine los diferentes enfoques metodológicos de corte humanistico-interpretativo y constructivista-cualitativo. Algunos autores emplean estos términos en sus trabajos: Marshall y Rossman (1989), Lecompte, Millroy y Preisle (1992), Glesne y Peshkin (1992) o Denzin, y Lincoln (1994).

3 Se suele reconocer que Spencer, junto a Taylor y Malinowski, iniciaron con distintos matices el método comparativo, consistente en la «acumulación de costumbres e ideas recogidas en diferentes lugares y procedente de periodos muy distintos, a fin de justificar esquemas de desarrollo a los que se había llegado por una vía más especulativa» (Mair, 1978). En un intento de dotar de cientificidad a los estudios antropológicos, se fueron formulando variantes tales como la "comparación estructural» (Lévi-Strauss, 1975), la "com- 


\section{Los estudios de caso en la lógica de la investigación...}

paración controlada» (Eggan, 1975) o la "comparación constante» (Glasser y Strauss, 1967).

4 Para Spradley (1980, pp. 70-75), el análisis de datos es: el conjunto de manipulaciones, transformaciones, reflexiones y comprobaciones realizadas a partir de los datos con el fin de extraer significado relevante para un problema de investigación.

\section{Bibliografía}

Anguera, M. ${ }^{\text {a }}$ T. (1995a): Metodología cualitativa. En Métodos de investigación en Psicología (pp. 513-522). Madrid: Síntesis.

Anguera, M. ${ }^{\text {a }}$ T. (1995b): Recogida de datos cualitativos. En Métodos de investigación en Psicología (pp. 523-548). Madrid: Síntesis.

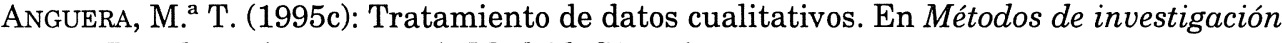
en Psicología (pp. 549-576). Madrid: Síntesis.

BARDín, L. (1986): El análisis de contenido. Madrid: Akal.

BARTOLOMÉ, M. (1992): La investigación cualitativa en educación: ¿Comprender o transformar? Revista de Investigación Educativa 20(2), 7-36.

BARTOLOMÉ, M. (1997): Panorámica general de la investigación sobre educación intercultural en Europa. Revista de Investigación Educativa 15(1), 7-30.

BARTolomé, M. et al. (1997): Una visión etnográfica. En Diagnóstico a la escuela multicultural, M. BARTOLOMÉ (Coord.) (pp. 191-296). Barcelona: Cedecs-Psicopedagogía.

Bogdan, R. y BIKLen, S. (1982): Qualitative research for education. Londres: Allyn y Bacon.

CoLÁs, P. (1994): La metodología cualitativa en España. Aportaciones científicas a la educación. Bordón 46(4), 407-421.

Coller, X. (2000). Estudio de casos. (Cuadernos metodológicos, n. ${ }^{\circ} 30$ ). Madrid: Centro de Investigaciones Sociológicas.

DenzIN, N. K. y LinColn, Y.S. (Eds.) (1994): Handbook of qualitative research. Londres: Sage.

ERICKSON, F. (1989): Métodos cualitativos de investigación sobre la enseñanza. En La investigación de la enseñanza, II. Métodos cualitativos y de observación, M. C. WITTROCK, (Ed.) (pp. 195-302). Barcelona: Paidós-MEC.

Fernández Cano, A. (1995). Métodos para evaluar la investigación en Psicopedagogía. Madrid: Síntesis.

García, E., GiL, J. y Rodríguez, G. (1994): Análisis de datos cualitativos en la investigación sobre la diferenciación educativa. Revista de Investigación Educativa 23(1), 183-201.

GIL Flores, J. (1994): Análisis de datos cualitativos. Aplicaciones a la Investigación Educativa. Barcelona: PPU.

Glasser, B. G. y Strauss, A. L. (1967): The discovery of grounded theory: Strategies for qualitative research. Chicago: Aldine.

Glesne, C. E. y Peshinin, A. (1992): Becoming qualitative researcher. An introduction. Nueva York: Longman.

Goetz, J. P. y Lecompte, M. D. (1988): Etnografia y diseño cualitativo en investigación educativa. Madrid: Morata.

GUBA, E. G. y LiNCOLN, Y. S. (1994): Competing paradigms in qualitative research. En Handbook of qualitative research, N. K. Denzin e Y. S. Lincoln (Eds.) (pp. 105-117). Londres: Sage. 
GUTIÉRREZ, J. (1999): El proceso de investigación cualitativo desde la investigación interpretativa y la investigación acción. En Modelos de análisis de la investigación educativa, L. Buendía, D. GonzÁlez, J. GutiÉrRez y M. Pegalajar (Eds.) (pp. 65-79). Sevilla: Alfar.

Hammersley, M. y Atkinson, P. (1994): Etnografia. Métodos de investigación. Madrid: Paidós.

Huberman, A. M. y Miles, M. B. (1994): Data management and analysis methods. En Handbook of qualitative research, N. K. Denzin e Y. S. Lincoln (Eds.) (pp. 429-444). Londres: Sage.

JANESICK, V. J. (1994): The dance of qualitative research design: Metaphor, methodolatry, and meaning. En Handbook of qualitative research, N. K. DENZIN e Y. S. LincolN (Eds.) (pp. 212- 234). Londres: Sage.

KRIPPENDORF, K. (1990): Metodología del análisis de contenido. Teoría y práctica. Barcelona: Paidós.

Lecompte, M. D., Millroy, W. L. y Preisle, J. (1992): The handbook of qualitative research in education. Nueva York: Academic Press.

LÉVI-STRAUSS, C. (1975): Las tres fuentes de la reflexión etnológica. En La antropología como ciencia, J. LloBera (Comp.) (pp. 15-24). Barcelona: Anagrama.

Lincoln, Y. S. y GuBA, E. G. (1985): Naturalistic inquiry. Beverly Hills, CA: Sage.

MAIR, L. (1978): Introducción a la Antropología Social. Madrid: Alianza.

Marshall, C. y Rossman, G. M. (1989): Designing qualitative research. Londres: Sage.

MAYkut, P. y MoRhouse, R. (1994): Beguinning qualitative research. A philosophic and practical guide. Londres: The Falmer Press.

MERRIAM, S. (1998): Qualitative research and case study applications in Education. San Francisco: Jossey-Bass.

Miles, M. B. y Huberman, A. M. (1984): Qualitative data analysis: A sourcebook of new methods. Newbury Park, CA: Sage.

PANOFF, M. F. (1975): ¿Para qué sirve la etnografía? En La antropología como ciencia, J. LlOBERA (Comp.) (pp. 79-82). Barcelona: Anagrama.

PÉrez Serrano, G. (1994): Investigación cualitativa. Retos e interrogantes. I-II. Madrid: La Muralla.

RAGIN, C. (1987): The comparative method. Moving beyond qualitative and quantitative strategies. Berkeley: University of California Press.

RAGIN, C. y BECKER, H. (1992): What is a case? Exploring the foundations of social inquiry. New York: Cambridge University Press.

Rodríguez, G., GiL, J. y GaRcíA, E. (1996): Métodos de investigación cualitativa. Málaga: Aljibe.

Rodríguez, G., GiL, J. y García, E. (1995): Análisis de datos cualitativos asistido por ordenador: AQUAD y NUDIST. Barcelona: PPU.

Ruiz OlabuÉnaga (1996): Metodología de la investigación cualitativa. Bilbao: Universidad de Deusto.

SÁNCHEz CARRIÓN, J. J. (1984): Técnicas de análisis de los textos mediante codificación manual. Revista Internacional de Sociología 43(1), 89-118.

SPRADLeY, J. P. (1980): Participant observation. Nueva York: Rinehart y Winston.

STAKE, R. E. (1998): Investigación con estudio de casos. Madrid: Morata.

TAYLOR, S. J. y BogDAN, R. (1984): Introducción a los métodos cualitativos de investigación. Buenos Aires: Paidós.

TEsh, R. (1990): Qualitative research: Analysis and software tools. Bristol: The Falmer Press. 


\section{Los estudios de caso en la lógica de la investigación...}

Velasco, H. y Díaz, A. (1997): La lógica de la investigación etnográfica. Valladolid: Trotta.

UniversidAD DE ChICAGo (1993): The Chicago manual of style (14.. ed.). Chicago: Chicago University Press.

WALFORD, G. (1995): La otra cara de la investigación educativa. Madrid: La Muralla.

YIN, R. K. (1989): Case study research: Design and methods (ed. rev.). Newbury Park, CA: Sage. 